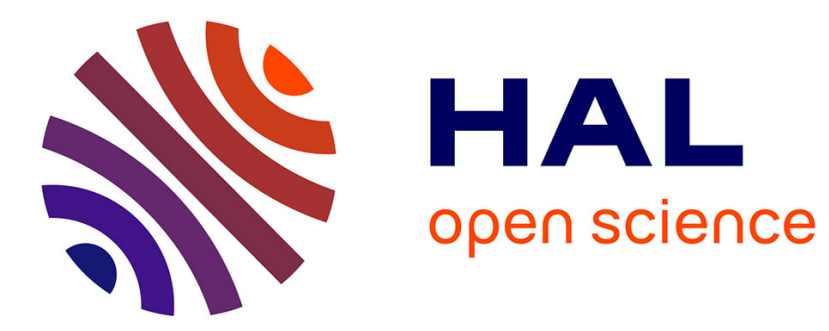

\title{
Transidentités et changement de sexe: le point de vue du sociologue - le rôle du psychiatre
}

\author{
Arnaud Alessandrin, Thierry Gallarda, Jean-Pierre Bouchard
}

\section{To cite this version:}

Arnaud Alessandrin, Thierry Gallarda, Jean-Pierre Bouchard. Transidentités et changement de sexe: le point de vue du sociologue - le rôle du psychiatre. Annales Médico-Psychologiques, Revue Psychiatrique, 2019. hal-02290302

\section{HAL Id: hal-02290302 \\ https://hal.science/hal-02290302}

Submitted on 17 Sep 2019

HAL is a multi-disciplinary open access archive for the deposit and dissemination of scientific research documents, whether they are published or not. The documents may come from teaching and research institutions in France or abroad, or from public or private research centers.
L'archive ouverte pluridisciplinaire HAL, est destinée au dépôt et à la diffusion de documents scientifiques de niveau recherche, publiés ou non, émanant des établissements d'enseignement et de recherche français ou étrangers, des laboratoires publics ou privés. 


\title{
Entretien
}

\section{Transidentités et changement de sexe : le point de vue du sociologue, le rôle du psychiatre}

Trans identity and sex change: The sociologist's point of view, the psychiatrist's role

\author{
Arnaud Alessandrin ${ }^{\mathrm{a}}$, Thierry Gallarda ${ }^{\mathrm{b}}$, Jean-Pierre Bouchard ${ }^{\mathrm{cd}}$ \\ ${ }^{\mathrm{a}}$ Laboratoire Cultures-Éducation-Sociétés (LACES EA 7437), faculté de sociologie, université de Bordeaux, 3 ter, place de la Victoire, 33076 Bordeaux Cedex, France \\ b "Consultation dysphorie de genre », Hôpital Sainte-Anne, Groupe Hospitalier Universitaire (GHU) Paris Psychiatrie et Neurosciences, 1, rue Cabanis, 75674 Paris cédex 14, \\ France \\ c Institut Psycho-Judiciaire et de Psychopathologie (IPJP), Institute of Forensic Psychology and Psychopathology, centre hospitalier de Cadillac, 10, avenue Joseph-Caussil, \\ 33410 Cadillac, France \\ Unité pour malades difficiles (UMD), pôle de psychiatrie médico-légale (PPML), centre hospitalier de Cadillac, 10, avenue Joseph-Caussil, 33410 Cadillac, France
}

\section{Introduction}

Qu'entend-on par transidentités ? Pourquoi des personnes transidentitaires ne veulent pas ou veulent changer de sexe ? Quel est le rôle du psychiatre dans le changement de sexe de personnes transidentitaires ? C'est à ces questions, aux enjeux souvent complexes, qu'Arnaud Alessandrin, sociologue, et Thierry Gallarda, psychiatre, répondent dans cet entretien.

Arnaud Alessandrin est sociologue à l'université de Bordeaux (Laboratoire Cultures-Éducation-Sociétés/LACES) où il enseigne la sociologie du genre et des discriminations. Après avoir soutenu en
2012 une thèse de sociologie intitulée « Du 'transsexualisme' aux devenirs trans », il développe de nombreuses recherches autour des discriminations LGBT, des questions de santé, notamment en cancérologie, et des questions d'espace public. Avec Johanna Dagorn, il coordonne la revue Les cahiers de la LCD depuis 2016. Il a notamment publié Sociologie des transidentités (Cavalier Bleu éd., 2018) et Parcours de santé-Parcours de genre avec Anastasia Meidani (PUM, 2018).

Thierry Gallarda est psychiatre, psychothérapeute, chef de service au pôle 16 de l'hôpital Sainte-Anne au sein du Groupe Hospitalier Universitaire (GHU) Paris Psychiatrie et Neurosciences. Au côté de la psychologue Sandrine Coussinoux [27-32,39-41,43-47,61,65], il est à l'origine de la consultation «dysphorie de genre et santé mentale » mise en place dans cette institution depuis plus d'une vingtaine 
d'années au sein d'un réseau pluridisciplinaire de soins qui alliait alors la clinique des maladies métaboliques et endocriniennes de l'hôpital Cochin (Dr Catherine Brémont-Weill \& Pr J.-P. Luton), le départe-ment de chirurgie plastique de l'hôpital Saint-Louis (Pr Revol \& Pr Banzet) et le service de psychiatrie de l'hôpital Foch (Dr Bernard Cordier \& Dr Bérénice Gardel). Il est à l'initiative de la constitution d'un réseau parisien de soins en santé mentale à vocation régionale destiné aux personnes trans intitulé « Réseau transidentité Paris GHU » dont les acteurs principaux (Dr Catherine Doyen, Dr Jean Chambry, Dr Serge Héfez, Dr Sébastien Machefaux) offrent des soins spécialisés à tous les âges de la vie, de l'enfance à la maturité, en veillant aux conditions optimales de relais entre les professionnels de la psychiatrie infanto-juvénile vers ceux des équipes de psychiatrie adultes. Ce réseau de soins est à destination de toutes les personnes trans, avec ou sans cooccurrence avec des troubles mentaux et des handicaps psychiques et de leurs familles.

\section{Interview}

\subsection{Jean-Pierre Bouchard: Qu'entend-on par transidentités?}

Arnaud Alessandrin : Pour répondre à cette question, il faut faire un petit saut dans le temps. En 1953, l'endocrinologue Harry Benjamin fait adopter l'idée que les personnes qu'il nomme «transsexuelles » sont atteintes d'un trouble, distinct de l'homosexualité, qui s'enracine dès le plus jeune âge et qui se manifeste par un désir irrépressible de changer de sexe. Cette permanence de la demande associée à la souffrance qui doit en découler fonde la première définition du «transsexualisme » [16]. En disant cela, j'insiste sur le fait qu'avant cette date, il n'y avait pas, à proprement parler, de personnes «transsexuelles ». Une revue de la littérature nous indique d'ailleurs que les occurrences liées à ce concept naissent avec la définition proposée par Harry Benjamin. Avant, il était question de «transvestis » ou « d'éonistes ». Avec la conceptualisation d'Harry Benjamin, les personnes « transexuelles » passent du statut d'anormales à celui de personnes « malades ». Si cette catégorisation fait aujourd'hui bondir, à juste titre, ce glissement promettait à l'époque une prise en charge des demandes des patientes et des patients (le terme de patient apparaît lui-même pour partie aujourd'hui impropre). Si vous me le permettez, j'insisterai de nouveau sur ce point. Penser qu'il y a toujours eu des personnes «transsexuelles » est un anachronisme conceptuel. La vie de Lili Elbe, une des premières personnes à subir une opération de réassignation dans les années 1930, en est un témoignage flagrant [52]. En effet lorsque Lili Elbe est admise à l'hôpital pour son opération (elle en subira en réalité plusieurs, dont une greffe d'utérus dont elle décédera), elle est admise en tant que «patient homosexuel » demandant une «stérilisation». C'est dire combien les concepts et les définitions forgent non seulement les représentations mais également nos actions, en matière de santé et de prise en charge notamment [4].

Mais revenons à votre question autour des transidentités et à cette histoire que je vous contais. Le concept de «transsexualisme » se sédimente très vite après son acceptation clinique et, en France par exemple, dès 1956 on recense des thèses sur la question. La décennie qui suit est de nouveau marquée par une effervescence scientifique autour de ce thème mais la France se démarque d'autres pays, comme les États-Unis par exemple, par la présence excessivement forte des discours psychanalytiques qui, ailleurs, sont progressivement remplacés par les études psychiatriques, endocrinologues et chirurgicales pour décrire le « transsexualisme » [36]. Cette spécificité française aura, jusqu'à aujourd'hui, des conséquences sur la manière de prendre en charge les personnes trans. Si nous traversons
l'Atlantique, toujours dans les années soixante, les thèses autour du concept de «genre » se multiplient. Le cas Agnès, décrit par le sociologue Garfinkel [48], illustre le passage progressif de la question médicale du «transsexualisme » à la question «sociale». Il ne s'agit plus de savoir seulement «pourquoi » les personnes « transsexuelles » expriment un désir de changer de sexe, mais plus encore de savoir « comment » elles y parviennent, à l'encontre de normes et de traditions puissantes. C'est dans cet esprit que des « gender clinic » verront le jour aux États-Unis afin de répondre aux demandes des personnes concernées [35]. Un point de vocabulaire important : en réalité les personnes ne « changent » pas de genre. Elles deviennent qui elles sont !

La France reste très en retrait de ces débats. La question du genre, hier comme aujourd'hui, est très mal perçue dans l'Hexagone [15]. Il faut attendre le tout début des années, 1980-1981 pour être précis, pour que la Sécurité sociale entérine le premier protocole de changement de sexe (Paris, Lyon, Bordeaux et Marseille en bénéficieront). Avant 1981, l'opération de changement de sexe était considérée par la caisse primaire d'assurance maladie (CPAM) et l'ordre des médecins comme une mutilation [2]. Dans ce contexte, les opérations avaient souvent lieu à l'étranger, notamment dans la très controversée clinique du professeur Burou à Casablanca [12] ! Après 1981, des protocoles sont donc instaurés. Le suivi protocolaire est fixé de la sorte : la psychiatrie diagnostique le «transsexualisme » de la personne, avant de l'envoyer vers un endocrinologue puis un chirurgien. Notons à cet égard qu'il n'y a pas, véritablement, de diagnostic du « transsexualisme ». Il s'agit d'un diagnostic différentiel qui vise à écarter les demandes qui prendraient la forme du «transsexualisme » sans en être, comme les troubles schizophréniques. Dans les écrits des équipes protocolaires, cette étape psychiatrique vise à délimiter une frontière entre de « vrais » et de «faux » patients. Elle vise aussi à limiter ce qui relèverait du « regret », à savoir les personnes qui désireraient une nouvelle fois changer de sexe après l'opération. Une revue de la littérature quelque peu sérieuse nous permet de constater que, en moyenne, $1 \%$ des personnes prises en charge émettent des regrets post-opératoires. Mais à la lecture de ces « regrets » il convient de noter que ces derniers ne renvoient pas au changement en tant que tel, mais plus à la technique opératoire ou bien encore au suivi médical lui-même [3,11] ! Insistons encore un peu sur ce point. Du point de vue médical, cette étape de tests psychiatriques est pensée comme un principe de précaution à visée thérapeutique [23]. Du point de vue sociologique, cela interroge fortement les notions d'éthique [6] et de discriminations. Ainsi, la reconnaissance et le remboursement de ces opérations sur le sol français dès 1981 ne sont pas sans conséquence. Certes, les personnes sont dorénavant prises en charge sur le modèle diagnostique proposé par Harry Benjamin 28 ans auparavant. Mais les critères de prise en charge sont à ce point stricts que le nombre de personnes inscrites en file active auprès d'endocrinologues ou de psychiatres hospitaliers est démesurément supérieur au nombre de personnes opérées. Pour reprendre les termes d'Alain Giami, l'offre de soins ne répond pas à la demande [49].

Les années 1990 sont quant à elles marquées par un triple phénomène, un triple débordement du concept et des représentations associées au «transsexualisme ». Tout d'abord, les personnes trans finissent par se rassembler. À l'image des personnes homosexuelles, la structuration associative et communautaire permet une déprise de l'isolement, de la honte, du tabou. Elle permet également des échanges d'expériences, des comparaisons entre les parcours, entre les prises en charges et développe une entre-aide bienvenue. Assez rapidement, Internet aidant, les associations trans se multiplient et se transforment jusqu'à devenir des espaces de contre-expertises face 
aux discours médicaux et des interfaces politiques autour des notions de genre [26] ou de lutte contre la transphobie [9] par exemple.

Deux autres débordements accompagnent cela. Le premier renvoie aux avancées juridiques qui, après de multiples condamnations de la France par la Cour européenne de Justice, octroient aux personnes trans des droits qui jusque-là leur étaient refusés. Entre autres exemples, on peut souligner qu'avant 2016 la transphobie n'était pas, en tant que telle, reconnue dans le droit français [7]. Dans un même mouvement, toujours avant 2016, les personnes trans devaient faire la preuve d'une "irréversibilité » de l'opération pour obtenir un nouvel état civil. Cette notion d' « irréversibilité » était entendue par les tribunaux comme une stérilisation. Cela signifie qu'un homme trans ne pouvait garder son utérus et, inversement, qu'une femme trans ne pouvait garder son pénis. Plus encore, les gamètes des personnes n'étaient pas préservés avant l'opération (et ne le sont d'ailleurs pas toujours), alors même que dans des cas similaires, comme lors d'un cancer des testicules, les gamètes des patients sont préservés. Cette exigence de stérilisation rendait, à proprement parler, la vie des personnes trans invivable car que fait-on sans un état civil qui correspond à notre genre ? En ce sens, la prise en charge du « transsexualisme » se heurte aux principes de lutte contre les discriminations et les équipes hospitalières réunies autour de la SOFECT (Société Française d'Étude et de prise en Charge du Transsexualisme) sont régulièrement prises pour cible à cet égard [4].

Un troisième débordement doit enfin être souligné, un débordement sémantique. Avec l'essor de la théorie « queer » [20] et des mouvements LGBT — lesbiens, gays, bissexuels et trans — les identités de genre et de sexualité fourmillent [8]. L'hétérosexualité et la cisidentité (le fait de ne pas vouloir changer de genre [63]) ne sont plus des modèles normatifs aussi forts. Ou plutôt, ils tendent à s'effriter. Lentement mais assez inéluctablement. Ainsi, en 2015, nous établissions la première mesure statistique de la transphobie en France et que nous demandions aux personnes concernées «quel est votre genre ? ", nous avions obtenu près de 36 réponses différentes. Hommes, femmes, trans, hommes trans, transsexuel. le. s, bigenre, agenre... [33]. L'explosion des identités signe un éloignement brutal entre les assignations binaires (femme-homme), les expressions et les expériences de genre [60]. Plus encore, en 2018, lorsque nous rendions publics les chiffres relatifs à la recherche "santé LGBTI », mes collègues et moi trouvions 42 identités de genre différentes à cette même question : " quel est votre genre ? ». Nous pourrions en lister quelques-unes : agenre, bigenre, gender fluid, transgenre, FtM (Female to Male), MtF (Male to Female), transsexuel. le, travesti. e, queer... [3]. C'est dire combien, pour les personnes trans, le modèle « transsexuel » d'hommes «qui deviennent» des femmes et inversement est un modèle qui tend vers l'obsolescence en ce sens qu'il ne répond que très partiellement aux demandes de prise en compte contemporaines [10].

Au total, le «transsexualisme » est peu à peu remplacé par des façons d'être soi, dans son sexe comme dans son genre, que le terme parapluie « transidentités » ou «personnes trans » traduit avec plus d'inclusion et moins d'épaisseur pathologisante [34]. D'ailleurs, les nomenclatures comme le DSM épousent ce glissement vers une reconnaissance de genre plus fluide et une pathologisation moindre [5].

\subsection{Jean-Pierre Bouchard: Pourquoi des personnes transidentitaires} ne veulent pas ou veulent-elles changer de sexe?

Arnaud Alessandrin : La question du «pourquoi » est particulièrement intéressante car elle relève, selon moi, d'une aporie, c'està-dire d'une question sans réponse. Pourtant, jusqu'à aujourd'hui, la médecine a tenté de répondre à cette interrogation : qu'est-ce qui pousse des individus à vouloir changer de sexe (ou de genre puisque les personnes trans opérées restent minoritaires parmi les transidentités, y compris parmi les personnes qui se disent ellesmêmes « transsexuelles »). Reprenons notre fil historique et regardons ensemble les « raisons » au changement de sexe, invoquées par les différentes sciences depuis 60 ans maintenant. À la toute fin des années 1950, deux thèses sont publiées, celle d'Alby [1] et celle d'Israel et Geissmann [38]. Pour ces auteurs, la psychanalyse seule est à même de traiter les demandes des personnes car la chirurgie ne répondrait qu'aux symptômes. Dans ces travaux, l'étiologie du

«transsexualisme » trouve sa source dans des troubles hystériques et obsessionnels et dans une forme extrême d'homosexualité. Si l'on pourrait croire que ces publications sont d'un autre âge, des publications plus récentes tendent à prouver que cette lecture psychopathologisante des transidentités demeure dans certaines pratiques cliniques. Ainsi, Colette Chiland [22] ou Henry Frignet [32] développeront eux aussi l'idée que cette demande, qu'ils considèrent comme anormale, doit être contenue et que la psychanalyse est en mesure de répondre aux demandes de transition. À cet endroit du débat, Colette Chi-land assumera même publiquement ses désaccords avec la prise en charge médicochirurgicale $\mathrm{du}$ «transsexualisme » [21]. C'est très certainement cette acception du « transsexualisme » qui a le plus marqué les protocoles de prise en charge, les pratiques cliniques [35] et les représentations sociales et médiatiques, même si ces dernières tendent toutefois à se modifier. Soulignons enfin que pour ces auteurs, auxquels nous pourrions ajouter Patricia Mercader [5], les discours des personnes trans sont jugés comme suspects : soit trop prévisibles, soit trop militants, soit trop stéréotypés. Face au fourmillement identitaire et à la réalité des actes transphobes, un regard sociologique sur ces textes ne peut être que frappé par la dimension profondément normative de ces écrits. De très nombreux auteurs convoquent d'ailleurs les mêmes critiques à l'égard de ce courant de pensée, que ce soit en Anthropologie (Laurence Herault [51]), en Psychologie (Françoise Sironi [64]) ou en Psychanalyse (Thamy Ayouch [12]).

Une deuxième réponse à la question du «pourquoi » a tenté d'être développée en France, sans succès à vrai dire, autour des recherches en neurobiologie. C'est le psychiatre Marc Louis Bourgeois [18,19] qui aura poussé le plus loin les importations de recherches américaines en France. Toutefois, ni aux États-Unis ni ailleurs ces recherches ont donné des résultats probants. Les inextricables controverses actuelles autour de la plasticité du cerveau face aux contraintes et aux injonctions de genre nous donnent une mesure de l'impasse dans laquelle se trouvent ces tentatives d'explications.

Une troisième réponse se borne, quant à elle, à des explications environnementales. On retrouve les prémices des explications dans les thèses de Robert Stoller [66] qui considère que des «quantités » de masculin ou de féminin peuvent affecter le développement de l'enfant in utero et lors du développement de son identité de genre au sein de la famille. Les rôles genrés des parents sont alors mis à contribution pour expliquer, à l'instar de ce qui était jadis fait pour l'homosexualité, des expressions de genre inhabituelles. Des auteurs plus contemporains comme Colette Chiland, Henry Frignet, Mireille Bonierbale ou Nadine Grafeille [50] considèrent que les demandes de changement de sexe s'apparentent à de la "médiagenèse », des phénomènes de mode, qu'il convient de suspecter fortement. L'environnement médiatique aurait, pour ces analystes, une influence sur le nombre de demandes de changement de sexe. On ne peut nier que les environnements familiaux ou culturels aient une influence sur les expressions transidentitaires. Mais le sociologue que je suis traduirait cela bien différemment. Dans un contexte de plus grande visibilité et acceptation des transidentités, comme cela fut le cas pour l'ho 
mosexualité, les individus acceptent de se penser et de se prononcer dans les termes de la transidentité !

C'est là une quatrième manière de répondre à la question du « pourquoi ». Il n'y a plus, aujourd'hui, d'identité à l'unité robuste et définitive. L'identité de genre ne fait pas exception. Ce qui résiste à l'émiettement complet des récits de vie c'est la façon dont les individus contemporains réinjectent de la cohérence. Cette cohérence, elle est aujourd'hui singulière, individuelle. Elle se dérobe aux grandes classifications. Le «pourquoi » du changement de sexe et du changement de genre ne réside donc très certainement plus dans des explications traumatiques ou environnementales mais dans l'explosion des identités ou, pour le dire autrement, dans leur détraditionnalisation [54].

\subsection{Jean-Pierre Bouchard: Quel est le rôle du psychiatre dans le changement de sexe de personnes transidentitaires?}

Thierry Gallarda : Le sexe renvoie aux caractéristiques biologiques, physiologiques, qui différencient les hommes et les femmes, alors que le genre renvoie davantage à la dimension sociale et culturelle de la sexuation : les rôles, les comportements, tous les attributs qu'une société considère à un temps donné comme appropriés à un sexe. Le « rôle de genre », c'est la face publique et sociale, «l'identité de genre » est la face plus privée, plus psychologique, la manière dont chaque personne vit son identité [25]. On peut vivre son identité sexuée de manière très diverse sans éprouver de malaise particulier.

Le terme dysphorie de genre (DG) décrit le sentiment de malaise, de détresse ou de souffrance qui peut être exprimé parfois par les personnes dont l'identité de genre, l'identité sexuée, ne correspond pas au sexe qui leur a été assigné à la naissance. Ces personnes peuvent s'identifier comme présentant une variance, de genre ou bien comme trans, transgenre, transsexuel-le-s, transvestis, « queer », androgynes, neutres, ou autre.

Parfois la détresse peut être si forte que certaines personnes s'engagent dans un processus de transition d'un sexe à l'autre afin d'établir une concordance et une continuité entre le sexe physique ou anatomique et l'identité sexuée. Ce processus implique habituellement des changements au niveau du rôle et de la présentation sociale et peut éventuellement nécessiter la prise d'hormones virilisantes ou féminisantes, voire des actes chirurgicaux.

La consultation «dysphorie de genre et santé mentale » se compose d'une équipe de psychiatres et de psychologues spécialisés dans la prise en charge des enfants, des adolescents et des adultes de tous âges présentant une souffrance psychique en lien avec l'expression et/ ou l'affirmation de leur identité sexuée.

Cette souffrance peut donc être d'intensité variable. Certaines personnes éprouvent un inconfort avec l'appartenance sexuée qui leur a été assignée à la naissance ; d'autres éprouvent un malaise avec les rôles sexués requis par la société. Certaines personnes peuvent aboutir à s'identifier comme transgenre ou de genre non conforme, alors que d'autres aboutissent à s'identifier comme des garçons, des filles ou comme des personnes qui sont tout simplement différentes.

Les motifs pour lesquels une personne trans sollicite l'aide d'un professionnel en santé mentale sont divers, à l'aune de la diversité de profils de cette population $[39,40,42,46]$. Les motifs de consultation peuvent être uniques, circonscrits par exemple à une demande d'adressage à un chirurgien pour la réalisation d'un geste « soumis à avis psychiatrique préalable » (le plus souvent une chirurgie génitale) ou sensiblement plus généraux : par exemple, lorsque des personnes trans s'interrogent sur le bien-fondé d'une démarche de transition et sont désireuses qu'un professionnel les aide à cheminer dans leur réflexion ou encore lorsque ces personnes rencontrent d'importantes difficultés à assumer leur désir de transition au sein de leur couple ou de leur famille.

$\mathrm{Au}$ gré des consultations, avec l'approfondissement des situations individuelles et l'établissement d'un lien thérapeutique de qualité, ces motifs initiaux peuvent également se révéler à géométrie variable : tel adolescent, jeune adulte ou adulte plus âgé exprimant en première instance une plainte dysphorique s'autorisera ainsi à solliciter son interlocuteur sur son besoin d'être aidé dans d'autres secteurs de son fonctionnement s'il (si elle) a le sentiment que sa plainte principale a été accueillie et devrait être raisonnablement soulagée.

Ainsi, dans la mesure du possible, aux cours des premiers ren-dezvous, il est important pour le praticien de déterminer avec le consultant(e) le ou les motifs de consultation afin de faciliter au mieux l'orientation et/ou de structurer l'accompagnement et la prise en charge.

Du fait de l'identification de notre offre de soins comme une consultation de recours, au sein d'un réseau de soins pluridisciplinaire (« transparis »), un certain nombre de nos consultants s'adressent à nous en deuxième instance pour une évaluation et une orientation vers une thérapeutique médicale féminisante ou virilisante à plus ou moins brève échéance, d'autres sont à l'inverse des «primo-consultants ».

Dans le premier cas, les personnes ont effectué le plus souvent un parcours de soins préalable qui les a conduit(e)s auprès d'autres professionnels, dans des conditions et avec des destinées variables : certains de ces professionnels les auront eux-mêmes pris en soins jusqu'à un stade plus ou moins avancé de leur parcours, ils souhaitent un « deuxième avis » ou passer le relais, en particulier dans des situations s'avérant plus complexes que prévu; d'autres auront assumé de prime abord leur manque d'expérience pour mettre en œuvre cet accompagnement spécifique et les auront orientées vers des équipes spécialisées ; d'autres encore auront fait le choix de mettre en œuvre des mesures non spécifiques de soulagement de la souffrance psychique, dans l'attente d'une orientation spécifique...

Le motif de consultation des « primo-consultants » est généralement moins «arrimé » : du fait de leur jeune âge et de leur manque de maturité, du fait de la confrontation avec la concrétude d'une consultation médicale contrastant avec la virtualité des échanges sur les réseaux sociaux et le web, du fait d'une psychopathologie cooccurrente entravant l'expression et/ou la précision de la pensée ou encore du fait d'une position d'autocensure. Cette position est notamment à l'œuvre lorsque la crainte d'une déstabilisation de la dynamique conjugale ou familiale génère un vif sentiment de culpabilité chez le (la) consultant(e) ou que l'expression trop explicite par ce(tte) dernier(ère) d'un désir de transition auprès d'un professionnel de santé viendrait

«l'engager» de façon incontrôlée. De fait, il n'est pas rare que le désir de changement de sexe ne soit pas directement et spontanément abordé au cours de la première consultation.

Notre consultation est à la disposition :

- des personnes qui éprouvent le besoin de s'adresser à des professionnels de santé mentale pour partager leurs questionnements ou leurs préoccupations autour de l'identité sexuée [31,32] ;

- de celles qui souhaitent se renseigner auprès de ces professionnels sur les modalités du parcours médico-chirurgical de transition de sexe et/ou s'engager dans un tel parcours ;

- mais aussi d'un certain nombre dont les questionnements autour de l'identité sexuée ne constituent qu'une des facettes de questionnements identitaires et/ou existentiels plus larges [41,43-46]. 
De par son implantation au sein d'un GHU axé sur la santé mentale, elle accueille également les personnes souffrant de troubles mentaux ou de handicaps psychiques qui présentent une dysphorie de genre et s'interrogent et/ou sont désireux de s'engager dans une transition $[45,58,61]$. Enfin, avec l'assentiment des principaux concernés, il n'est pas rare que nous recevions des familles de jeunes adultes trans (parents le plus souvent, fratrie parfois) chez lesquelles la révélation de la transidentité d'un des enfants vient résonner avec des probléma-tiques individuelles, entraîne un besoin de «complément d'informa-tion » ou suscite une vive angoisse en lien avec une représentation négative et délétère des thérapeutiques de transition (toxicité des hor-mones, mutilation et stérilisation par les thérapeutiques chirurgi-cales...). Le choix du recours à une institution hospitalière publique est alors présenté à la fois comme celui d'un lieu empreint d'une plus grande neutralité et en capacité de proposer une offre de soins pérenne et dans la continuité [13]. La consultation «dysphorie de genre et santé mentale » a été mise à disposition des usagers par notre équipe

à l'hôpital Sainte-Anne voilà vingt-cinq ans [27,29,30]. Plus récemment, avec la création au $1{ }^{\text {er }}$ janvier 2019 du GHU Paris Psychiatrie et Neurosciences, notre expérience et nos pratiques ont été enrichies par celles d'autres professionnels de l'hôpital Sainte-Anne (Dr Catherine Doyen) et de l'EPS Maison Blanche (Dr Serge Hefez, Dr Jean Cham-bry). L'implantation de ces équipes et leurs compétences spécifiques (troubles du spectre autistique, adolescence, personnes concernées par le VIH, sexualité...) ont favorisé la structuration d'un réseau de soins dédiés aux personnes trans à tous les âges de la vie, avec ou sans antécédents de troubles mentaux.

Ces regards croisés ont facilité l'émergence d'une réflexion féconde sur l'évolution des pratiques médico-psychologiques auprès des personnes trans (cf. évolutions nosographiques des versions du DSM) au cours des trente dernières années. Au fil des ans, la médiatisation internationale des problématiques trans, les combats politiques menés par les mouvements associatifs auprès des autorités de santé (DGS, CNAM, HAS...) et/ou des sociétés savantes voire directement auprès de professionnels, accusés d'incarner tantôt une position d'omnipotence médicale, tantôt un paternalisme délétère et obsolète ont permis d'éclairer les questions transidentitaires d'un nouveau jour. Ce «work in progress » permanent conduit, contraint parfois, les professionnels à une évolution constante de leur réflexion autour de leurs cadres conceptuels et de leurs pratiques de soins [56].

L'évolution des pratiques a été intimement liée à la "dépathologisation » progressive des situations de transidentité, d'un modèle biopsychosocial commun à l'ensemble des troubles mentaux incarné dans les catégorisations nosographiques précédant le DSM-5 à celui d'une variance dimensionnelle du genre incarnée dans la notion de dysphorie et répondant à de multiples causes possibles, individuelles, environnementales, sociologiques.

C'est un profond changement de paradigme : nous sommes passés d'une approche biomédicale impliquant un relevé de symptômes (ou une « sémiologie ») [26], une anamnèse évocatrice, un diagnostic positif et l'élimination d'un ou de plusieurs diagnostics différentiels ouvrant l'accès à une option thérapeutique, à l'écoute et l'accueil attentifs d'une plainte, la «dysphorie » et à l'appréciation du contexte de son éclosion, de ses caractéristiques évolutives (épisodique, chronique), de son intensité, des stratégies mises en place spontanément par le (la) plaignant(e) pour la soulager et enfin de la nature, de l'étendue et de la recevabilité des demandes faites au corps médical pour l'apaiser.

Ce changement fondamental de paradigme a influé considérablement sur les pratiques en conférant une autonomie décisionnelle sensiblement plus importante aux personnes dysphoriques de genre ou, dit en termes éthiques, en déplaçant le curseur de la bi enfaisance et de la non-malfaisance vers l'autonomie [28,37]. De plus, en questionnant le binarisme des genres, l'intégration de la notion dimensionnelle inhérente à celle de la dysphorie a favorisé l'émergence de nouvelles populations trans, dites «non binaires » [60]. Sur le terrain, de cette diversité de populations découle naturellement une extension des possibilités d'adresse à la médecine et à la chirurgie, à la mesure des combinaisons psychiques possibles : par exemple, telle femme trans (male to female) n'envisage pas de vagino-plastie car elle n'éprouve pas de dysphorie de ses organes génitaux, tel homme trans (male to female) requiert une hystérectomie et une ovariectomie bilatérale associées à sa torsoplastie car il lui est inconcevable de « conserver » les organes génitaux le renvoyant à son sexe d'assignation, tel autre souhaitera à l'inverse conserver ses organes génitaux internes en circonscrivant sa transition à un traitement virilisant et à une chirurgie mammaire, telle femme trans demande en priorité une ablation de ses organes génitaux avec une féminisation hormonale a minima afin de se rapprocher au plus près de ses éprouvés identitaires...

Parmi les professionnels médicaux confrontés à cette créativité foisonnante dans la sculpture du corps au plus près des différentes « conceptions de soi », les psychiatres assument sans conteste une fonction et un rôle spécifique. Leur intervention est d'autant plus délicate qu'elle a pu être et demeure largement contestée du fait du mouvement de dépathologisation évoqué tout en demeurant incontournable dans l'offre actuelle de soins : facilitation de l'accès aux soins physiques de la transition (en particulier chirurgicaux) et à leur prise en charge par la Caisse Nationale d'Assurance Maladie (CNAM) mais surtout gestion des multiples expressions de la souffrance psychique venant compliquer la « dysphorie » ou associées à elle (anxiété, phobie scolaire et sociale, dépression, suicidalité, addictions, difficultés de communication intrafamiliales etc.) et accompagnement des «familles » (enfants, conjoints, parents).

Schématiquement, l'intervention des psychiatres peut se situer à différents niveaux, avec des temporalités différentes : au sein d'une équipe pluridisciplinaire et pluriprofessionnelle (endocrinologie, chirurgie, dermatologie, orthophonie...) lorsque la personne trans s'est engagée dans un parcours de transition, durant la période nécessaire à l'accomplissement de ce parcours, en amont de ce parcours et, dans certains cas, après la réalisation de ce dernier (ces missions apparaissent spécifiques et requièrent un savoir-faire et des compétences dans le champ des transidentités) ; en situation « hors parcours de transition », lorsque la personne trans souffre d'une altération de sa santé mentale, quelle que soit l'origine de cette altération [17] ; en cas d'insatisfaction voire de regret après que la transition a été effectuée. En effet, il faut souligner ici que les besoins généraux de santé des personnes trans sont les mêmes que ceux de tout le monde, incluant les besoins en santé mentale. La littérature souligne même que les personnes trans pâtissent d'une discrimination dans l'accès aux soins en comparaison avec la population générale. On rejoint dans ce cas la question du recours à des soins spécialisés en santé mentale que ces derniers soient prodigués à des populations cis ou à des populations trans. Nous restreindrons ici notre propos aux missions du psychiatre travaillant dans une équipe pluridisciplinaire.

De façon globale, l'évaluation par un psychiatre a pour objectif d'aider les personnes dysphoriques dans le genre qui leur a été assigné à la naissance à identifier le dispositif leur permettant de s'installer dans un rôle de genre plus acceptable. Cette évaluation a pour objectif de les accompagner dans la prise de décisions éclairées sur les interventions médicales disponibles et d'en « co-évaluer » avec le praticien les implications psychiques, sociales, profes 
sionnelles, sexuelles et légales. Autrement dit, face à une demande de changement de sexe, l'enjeu de l'évaluation d'une demande de changement de sexe sur un temps suffisant consiste à co-estimer avec le (la) demandeur(se) le rapport entre les bénéfices pouvant être attendus de manière réaliste et les risques potentiellement encourus lors d'une telle entreprise, à court, moyen et long terme.

L'appréhension du rapport à la réalité dans les domaines liés à la transition elle-même mais aussi dans les autres secteurs de fonctionnement de l'existence est un des facteurs pronostiques. Cette appréhension passe par la qualité de l'évocation et de la lecture que le(la) demandeur(se) fait de sa propre situation, personnelle, familiale, scolaire et/ou professionnelle et de ses attentes vis-à-vis de la transition. Des attentes « démesurées », naïves, quasi «magiques » en termes de modifications physiques comme en termes d'amélioration de fonctionnement répondent le plus souvent à une lecture initiale imprécise, floue ou en décalage avec la réalité et peuvent se solder par de sévères désillusions voire des regrets, précoces, dans le postopératoire immédiat ou plus tardifs. Les regrets émergeant dans le post-opératoire immédiat s'incarnent volontiers dans une insatisfaction liée aux résultats esthétiques ou fonctionnels qui n'était pas ceux qui étaient attendus, ils donneront lieu à des demandes de réinterventions correctrices à distance, validées ou questionnées par l'équipe chirurgicale. À plus long terme, les regrets apparaissent plus rares. Leur fréquence est estimée classiquement à $1 \%$ dans la littérature. Dans notre expérience, ils peuvent survenir chez des personnes trans aux personnalités pathologiques narcissiques que la transition ne soulage que partiellement d'une dépressivité et d'une mésestime de soi chronique et fondamentale.

Contrairement à certaines représentations communes, les populations trans se caractérisent par une grande diversité sociologique, culturelle, de leur statut médical... Cette diversité apparaît mieux prise en considération depuis les dernières années : l'extension de l'offre de soins autorise en effet la mise en œuvre d'un projet thérapeutique partagé, individualisé et plus satisfaisant, tant du point de vue des usagers que des praticiens.

Quel que soit l'âge du consultant, les entretiens avec un psychiatre répondent à plusieurs objectifs généraux. Certains objectifs sont facilement réalisables, d'autres moins.

Le premier objectif est de s'assurer avec la personne de la présence et de la stabilité des critères diagnostiques de la dysphorie de genre (DSM-5) de l'adolescent et de l'adulte. De notre expérience, il ressort que c'est le cas auprès de la grande majorité de nos consultants : les critères actuels de dysphorie de genre chez les adolescents et les adultes du DSM-5 sont largement inclusifs et leur stabilité communément observée lorsqu'ils sont retrouvés à ces âges de la vie (à l'inverse des dysphories exprimées dans l'enfance).

Un deuxième objectif est de s'assurer que la dysphorie de genre n'est pas secondaire ou mieux expliquée par d'autres conditions, en particulier un trouble mental, par exemple une schizophrénie avec des hallucinations corporelles portant sur les caractères sexuels primaires ou secondaires ou des idées délirantes de changement de sexe ou encore une manie avec symptômes psychotiques. Toujours dans notre expérience, ces situations de décompensation psychique avec expression d'une dysphorie de genre symptomatique dont la résolution s'accompagnerait d'une disparition totale de la dysphorie demeurent très rares $[17,47]$. Ce constat clinique mérite d'être souligné tant certains discours théoriques, psychiatriques et psychanalytiques autour du désir transsexuel comme symptôme psychotique ont pu être durablement véhiculés, entravant largement la qualité des prises en soins auprès de ces populations.

À l'inverse, les situations conjuguant un trouble mental et une dysphorie de genre cooccurrente «qui n'est pas expliquée par ce dernier » nous apparaissent sensiblement plus fréquentes [59]. Elles sont de facto surreprésentées à notre consultation sise au sein d'un établissement spécialisé en santé mentale, ce qui constitue un biais de recrutement. Ces situations relèvent aussi des missions du psychiatre. Elles requièrent une évaluation et une réflexion approfondies autour des parcours et des prises en soin.

Leur gestion optimale relève des compétences conjuguées d'une équipe spécialisée «dysphorie de genre et santé mentale » et de professionnels de santé mentale de proximité ou de praticiens de médecine générale sensibilisés à ces questions. L'implication de travailleurs sociaux et de personnels socio-éducatifs est également fréquente, en particulier chez les populations les plus jeunes et/ou les plus précaires.

Les compétences de l'équipe spécialisée sont requises à la fois dans l'évaluation diagnostique initiale et la conceptualisation du cas mais aussi dans le travail de coordination des différents acteurs en santé mentale et du médecin généraliste. Dans les cas où une transition de sexe est envisagée, ce travail de coordination s'étend aux autres acteurs impliqués dans les soins physiques.

Parmi les situations les plus fréquemment rencontrées : une personne, le plus souvent adolescent(e) ou jeune adulte souffrant d'un trouble du spectre autistique (le plus souvent un syndrome d'Asperger diagnostiqué ou non) et d'une dysphorie de genre venant majorer la souffrance psychique et l'altération du fonctionnement social préexistants ; une autre débutant un trouble affectif bipolaire ou traitée au long cours pour ce dernier dont la perspective d'une transition hormonale et/ou chirurgicale soulève la question d'un risque accru de décompensation du trouble de l'humeur à l'introduction des hormones ou lors de la phase chirurgicale ; celle d'une personne présentant une personnalité narcissique dont les attentes vis-à-vis des effets de la transition seront potentiellement déçues ou dont le fonctionnement antisocial (psychopathique) se plie très difficilement aux contraintes des parcours de soins pluridisciplinaires ; enfin, une personne trans souffrant d'un trouble obsessionnel compulsif (TOC) dont l'intensité des préoccupations obsédantes et/ou des rituels risquera d'être majorée en résonance avec certains éprouvés liés à la dysphorie (par exemple, idées obsédantes autour des zones de pilosité, rituels de lavage en réaction au dégoût des organes génitaux...).

Dans chacune de ces situations, souvent complexes au plan clinique, seule une évaluation minutieuse auprès d'un(e) clinicien(ne) permettra d'identifier au mieux la souffrance psychique et le retentissement liés au trouble mental de celle proprement en lien avec la dysphorie. De plus, la majorité de ces situations convoquent également la question de la co-prescription des psychotropes et des traitements hormonaux virilisants et féminisants au long cours. Les effets secondaires de certains psychotropes (par exemple, syndrome métabolique, gynécomastie, troubles du rythme cardiaque, troubles de la libido, troubles hépatiques...), en interférant avec ceux des hormones, imposent une surveillance médicale rapprochée.

$\mathrm{Au}$ sein de notre système de santé, les professionnels de santé mentale qualifiés dans le champ des dysphories de genre semblent également les mieux préparés à une évaluation des modalités d'ajustement psychosocial des personnes trans aux différents âges de la vie.

Dans l'enfance et à l'adolescence, la qualité de la relation aux pairs et de l'inscription dans le cursus scolaire peut être altérée par l'envahissement des préoccupations que le sujet a autour de sa dysphorie lorsqu'il a une conscience précoce de celle-ci, ou par un sentiment diffus « d'être différent ». L'altération provient également des réactions négatives que l'expression de la dysphorie risque de générer au sein du groupe ou de l'effort mental que le sujet consacre à la mas 
quer pour maintenir son intégration qui l'éloigne d'autant d'autres investissements.

En réponse à une exclusion active ou passive des groupes de pairs, aux railleries voire à des conduites de harcèlement, nos jeunes patients et leurs familles relatent ainsi la nécessité qui s'est imposée à eux de changements récurrents d'affectation scolaire ou leur choix forcé d'interrompre prématurément une scolarité devenue invivable en adoptant des modalités d'enseignement par correspondance ou en se lançant à corps perdu sans formation suffisante dans le monde du travail.

À l'âge adulte, la décision de s'engager dans une transition s'accompagne également d'un stress majeur en lien avec une anticipation négative des conséquences professionnelles et dans certains secteurs difficilement compatibles avec le déroulement d'une transition dans de bonnes conditions, de la nécessité d'une réorientation professionnelle.

Un accompagnement peut être particulièrement utile pour faciliter le processus de révélation ( « coming out») et/ou pour aborder le plus sereinement possible les éventuels remous institutionnels suscités par ce dernier. C'est le cas en particulier lors de l'installation des premières manifestations visibles de changement physique : distance ou proximité inhabituelles des collègues ou de l'encadrement, discriminations dans l'accès à des promotions, agressivité active (par exemple, mégenrage systématisé ou isolement forcé) ou passive, intimidations voire actes transphobes [65].

Enfin, l'appréhension d'éléments de la structure et de la dynamique familiale transgénérationnelle est essentielle. Quel que soit l'âge, l'existence d'un soutien familial exprimé sans ambiguïté est facilitante : parents, fratrie, conjoint, grands-parents aussi, notamment chez les adolescents et les jeunes adultes où l'accueil compréhensif de la dysphorie et d'un éventuel projet de transition a souvent valeur de médiation. Certaines demandes de transition suscitent des bouleversements tels au sein de couples ou de familles qu'une orientation en thérapie familiale peut être proposée.

\section{Déclaration de liens d'intérêts}

Les auteurs déclarent ne pas avoir de liens d'intérêts.

\section{Références}

[1] J.M. Alby, Contribution à l'étude du trans-sexualisme. Thèse de médecine, sous la direction de Jean Delay, Paris, 1956.

[2] A. Aguado, I. Zdanowicz, L'usage du droit dans le mouvement d'émancipation trans, Cah Genre 57 (2014) 77-94.

[3] A. Alessandrin, Le 'transsexualisme' : une nosographie obsolète, Sante Publique 24 (2012) 263-269.

[4] A. Alessandrin, K. Espineira, M.Y. Thomas, La SOFECT : du protectionnisme psychiatrique, Cah Transidentite 1 (2013) 61-75.

[5] A. Alessandrin, Ce que le DSM-5 fait aux variances de genre [en ligne], Socio-Logos 9 (2014).

[6] A. Alessandrin, Le principe de précaution est-il un principe éthique ?, Ethique Sante 11 (2014) 43-50.

[7] A. Alessandrin, La transphobie en France, Cah Genre 60 (2016) 193-211.

[8] A. Alessandrin, Pour en finir avec la notion de 3 sexe [en ligne], Socio 8 (2017).

[9] A. Alessandrin, Sociologie des transidentités, Cavalier Bleu éd, 2018.

[10] A. Alessandrin, Actualité des trans studies, AEC ed., 2019.

[11] A. Alessandrin, La notion de regret dans la clinique de changement de genre, Evol Psychiatrique 84 (2019) 277-285.

[12] Alessandrin A, Ouchen A. Une histoire des transidentités : échanges franco-marocains, in : Le genre entre France et Maroc (F. Borj dir.). Casablanca ed. [à paraître].
[13] I. Amado, T. Gallarda, Le syndrome de transsexualisme, in Troubles de l'orientation et de l'identité sexuelle. Entretiens de Bichat, Thérapeutique, Expansion Scientifique française, 1998277-280.

[14] T. Ayouch, Psychanalyse et transidentités : hétérotopies, Evol Psychiatrique 2 (2015) 303-316.

[15] B. Bellebeau, A. Alessandrin, Genre ! L'essentiel pour comprendre, Des ailes sur un tracteur, 2014

[16] H. Benjamin, Transvestism and transsexualism, Int J Sexol 7 (1953) 12-14.

[17] C. Berthon, T. Gallarda, S. Coussinoux, E. Rari, J.P. Olié, Demande de transformation hormono-chirurgicale du genre et transition psychotique à l'adolescence : à propos d'une observation, Encephale 32 (2006) 125.

[18] M.L. Bourgeois, Les traitements psychologiques du transsexualisme des troubles de l'identité sexuelle. Quelques tentatives récentes, Ann Med Psychol 136 (1978) 985-1008.

[19] M.L. Bourgeois, Transsexualisme, dysphorie de genre et troubles de l'identité sexuelle. Une expérience américaine, Ann Med Psychol 138 (1980) 472-480.

[20] J. Butler, Trouble dans le genre, La découverte, Paris, 2005.

[21] P.H. Castel, La métamorphose impensable, Gallimard, Paris, 2003.

[22] C. Chiland, Le transsexualisme, PUF « Que sais-je ? », Paris, 2003.

[23] C. Chiland, B. Cordier, Transsexualisme, EMC Psychiatrie, 20001-11.

[24] E. Coleman, W. Bockting, M. Botzer, et al., Standards of care for the health of transsexual, transgender, and gender-nonconforming people, version 7, Int J Transgenderism 13 (2012) 165-232.

[25] L. Compère, E. Rari, T. Gallarda, A. Assens, M. Nys, S. Coussinoux, et al., Gender identity better than sex explains individual differences in episodic and semantic components of autobiographical memory and future thinking, Conscious Cogn 57 (2018) 1-19.

[26] B. Cordier, C. Chiland, T. Gallarda, Le transsexualisme : proposition d'un protocole malgré quelques divergences, Ann Med Psychol 159 (2001) 190-195.

[27] S. Coussinoux, Analyse d'aptitudes cognitives différenciées selon le sexe chez des sujets transsexuels primaires, avant et après traitement hormonal, DEA de Psychopathologie et Neurobiologie des Comportements, Université Paris V, 1994.

[28] S. Coussinoux, J. Smith, T. Gallarda, J.P. Olié, Actualités éthiques sur le transsexualisme, "Repères", Forum, Espace Ethique Mediterraneen 9 (2004) 12-13.

[29] S. Coussinoux, I. Amado, T. Gallarda, B. Cordier, M.F. Poirier, J.P. Olié, As-pects neuropsychologiques du transsexualisme avant et après hormonothérapie, Réunion conjointe de l'AFPB et du Belgium College of Neuropsychopharmacol-ogy and Biological Psychiatry, Encephale XXII (1996) 303-304.

[30] S. Coussinoux, I. Amado, T. Gallarda, B. Cordier, C. Bremont, J.P. Luton, et al., Aspects neuropsychologiques du transsexualisme : résultats d'une étude préliminaire. L'encéphale, ${ }^{\circ}$ spécial, La Psychopathologie peut-elle être cognitive ?, Recherches Actuelles 21 (1998) 20-21.

[31] S. Coussinoux, T. Gallarda, B. Cordier, M. Haddou, I. Amado, M.F. Poirier, et al., M-F and F-M transsexuals: the value of personality tests, Eur Psychiatry 15 (1) (2000) 96-97.

[32] S. Coussinoux, T. Gallarda, J. Smith, M.C. Bourdel, B. Cordier, C. Brémont, et al., Tests de personnalité et identification sexuée chez des transsexuels masculins, Encephale 31 (2005) 24-30, [cahier 1]

[33] J. Dagorn, A. Alessandrin, La santé des LGBTI à l'école, Ecole Parents 627 (2018) 28-29.

[34] K. Espineira, M.Y. Thomas, A. Alessandrin, Transidentités : histoire d'une dépathologisation (dir.), Cah Transidentite (2013) 1.

[35] N. Fisk, Gender dysphoria syndrome: the conceptualization that liberalizes indications for total gender reorientation and implies a broadly based multidimensional rehabilitative regimen (Editorial comment on male transsexualism), West J Med 120 (1974) 386-391.

[36] M. Foerster, Histoire des transsexuels en France, H\&O ed, 2005.

[37] V. Fournier, Faut-il opérer les transsexuels ?, Le bazar bioéthique - Quand les histoires de vie bouleversent la morale publique, Robert Laffont, Paris, 2010.

[38] H. Frignet, Le transsexualisme, DDB, 2003.

[39] T. Gallarda, I. Amado, S. Coussinoux, B. Cordier, J.P. Olié, Le syndrome de transsexualisme : aspects cliniques et perspectives thérapeutiques, Encephale XXIII (1997) 321-326.

[40] T. Gallarda, Eirini Rari, S. Coussinoux, J. Smith, Les demandes de changement de sexe : questionnements éthiques émergeant d'une pratique clinique, Odile Bourguignon éd, Ethique et pratique psychologique, Mardaga, Wavre (Belgique), 2008175-187.

[41] T. Gallarda, S. Coussinoux, B. Cordier, M. Haddou, I. Amado, M.F. Poirier, et al., Épreuves de personnalité et représentations sexuées dans une cohorte française de sujets consultant pour des troubles de l'identité sexuelle, Encephale 26 (2000) 8081.

[42] T. Gallarda, Le syndrome de transsexualisme, in Séminaire de Psychiatrie biologique, Séminaire de Psychiatrie Biologique, Hôpital Sainte-Anne, 30, Aventis, 2000151-173 
[43] T. Gallarda, S. Coussinoux, B. Cordier, M. Haddou, I. Amado, M.F. Poirier, et al., Personality tests assesment in a selected cohort of french applicants for sex reassignement linked to the problem of sexual identity, Eur Psychiatry 15 (Suppl 2) (2000) $320 \mathrm{~S}$.

[44] T. Gallarda, S. Coussinoux, B. Cordier, M.C. Bourdel, J.P. Olié, Intérêt des tests de personnalité dans le diagnostic différentiel transsexualisme M-F/transvestisme, Ann Med Psychol 159 (2001) 466-470.

[45] T. Gallarda, J. Smith, N. Rahmani, S. Coussinoux, A. Sacco, M.C. Bourdel, J.P. Olié, Psychiatric and socio-demographic characteristics of 40 surgical sex-reassignment applicants, Int J Neuropsychopharmacol 7 (suppl. 1) (2004) P02539, [S 446].

[46] T. Gallarda, J. Smith, S. Coussinoux, Troubles de l'identité de genre, Encephale 31 (2005) 65-67, [cahier 2].

[47] T. Gallarda, S. Machefaux, S. Rari, S. Coussinoux, Le point de vue in Régis Mache (éd.), La nature dans l'identité sexuelle, L'Harmattan, Paris, 2009.

[48] H. Garkinkel, Recherches en ethnométhodologie, PUF, Paris, 2007.

[49] A. Giami, E. Beaubatie, J. Le Bail, Caractéristiques sociodémographiques, identifications de genre, parcours médicopsychologique et VIH/sida dans la population trans, BEH 42 (2011) 433-437.

[50] N. Grafeille, Prise en charge du transsexualisme en 2010 : l'expérience bordelaise, La transidentité (Alessandrin dir.), L'Harmattan, Paris, 201039-49.

[51] L. Herault, La parenté transgenre, PUP, 2014.

[52] N. Hoyer, Man into woman: an authentic record of a change of sex/Lili Elbe; edited by Niels Hoyer [i.e. E. Harthern]; translated from the German by H.J. Stenning; introd. by Norman Haire, Jarrold Publisher's, London, 1933.

[53] L. Israel, P. Geissmann, Le désir de changer de sexe chez les invertis psychosexuels, Cah Psychiatr 14 (1960) 91

[54] A. Meidani, A. Alessandrin, Cancers et transidentités : une nouvelle «population à risques ? », Sci Soc Sante 35 (2017) 41-63.

[55] P. Mercader, L'illusion transexuelle, L’Harmattan, Paris, 2000.
[56] F.L. Meyer-Bahlburg Heino, « Diagnosing » gender? Categorizing gender-identity variants in the anthropocene, Arch Sexual Behav (2019) https://doi.org/10. 1007/s10508-018-1349-6.

[57] A. Michel, C. Mormont, Transsexualisme et Rorschach : une revue de la littérature, Ann Med Psychol 161 (2003) 125-131.

[58] F. Mouaffak, T. Gallarda, N. Baup, J.P. Olié, M.O. Krebs, Gender identity disorders and bipolar disorder associated with the ring Y chromosome, Am J Psychiatry 164 (2007) 1122-1123.

[59] National LGBT health education center, Caring for transgender people with severe mental illness. A program of the fenway institute, in: https://www. lgbthealtheducation.org/wp-content/uploads/2018/06/Understanding-andAddressing-Severe-Mental-Illness-in-Transgender-People.pdf.

[60] F. Poirier, A. Condat, L. Laufer, et al., Non-binarité et transidentités à l'adolescence, Neuropsychiatr Enfance Adolesc 66 (6) (2018) https://www.sciencedirect. com/science/article/pii/S0222961718301211.

[61] N. Pommepuy, T. Gallarda, S. Coussinoux, A. Gut-Fayand, J. Smith, J.P. Olié, In: Demandes de réassignation hormono-chirurgicale du sexe et troubles mentaux : à propos d'un cas de transvestisme, L'Encéphale, Actes du Congrès, Acanthe-Masson, Paris, 2003, p. 165.

[62] A. Sacco, J. Smith, R. Bobet, T. Gallarda, J.P. Olié, In: Rorschach et troubles de l'identité de genre, L'Encéphale, Actes du congrès 2004, Acanthe-Masson, Paris, 2004, pp. 227-228.

[63] J. Serano, Whipping Girl, Seal Press, 2007.

[64] F. Sironi, Psychologie(s) des transsexuels et des transgenres, Odile Jacob, Paris, 2011.

[65] J. Smith, S. Coussinoux, T. Gallarda, J.P. Olié, In: Psychothérapie de groupe pour des patients demandeurs d'un changement de sexe, L'Encéphale, Actes du congrès 2004, Acanthe-Masson, Paris, 2004, p. 228.

[66] R. Stoller, A contribution to the study of gender identity, Int J Psychoanal 45 (1964) 220-226. 